\title{
Role of fine needle aspiration cytology in effective management of patients with breast lesions and histopathological correlation
}

\author{
Susmitha N.S ${ }^{1, *}$, Chaithra. $H^{2}$, Rajatha Anand ${ }^{3}$ \\ Assistant Professor, Dept. of Pathology, Sri Siddhartha Medical College, Tumkur, Karnataka, India
}

*Corresponding Author:

Email: sush_susmitha2000@yahoo.co.in

\begin{abstract}
Introduction: Increased incidence of breast cancer has evoked profound interest in benign breast lesions, since certain benign lesions show malignant transformation. Main objective of FNAC is to diagnose malignant lesions that require surgical excision from benign ones.

Aims and Objectives: The present study was carried out to know the scope of FNAC in accurately diagnosing various breast lesions and its role in effective management of patients.

Materials and Methods: A prospective study was conducted from September 2011 to Sept 2013 over duration of 24 months. 155 patients with breast lesions were subjected for FNAC and correlated with histopathological examination in 52 cases.

Results: Out of 155 FNACs done, cytohistopathological discordance was noted in 3 cases. An occasional false negative and false positive in disease were noted. Overall sensitivity, specificity, positive predictive and negative predictive value were $93.1 \%$, $97.1 \%, 96.4 \%$, and $94.4 \%$ respectively.

Conclusion: The accuracy of FNAC when combined with clinical examination and radiology is notably high. Since the majority of patients have benign disease, a rapid diagnosis and discharge is beneficial. The present study concluded that accuracy of FNAC in diagnosing various breast lesions is high however an occasional false negative and false positive cases makes it necessary to biopsy before mastectomy in cases where clinical, cytological and radiological diagnosis do not concur.
\end{abstract}

Keywords: FNAC, Breast lumps, Malignant, Benign.

\section{Introduction}

FNAC has been used extensively over the past 25 years. It plays a major role as an important preoperative assessment procedure along with clinical correlation and imaging which are referred to as the Triple test. ${ }^{1}$ Limitation of an FNAC is its inability to separate in situ and invasive carcinoma. ${ }^{2}$

Breast lesions are the most common lesions seen in our patients. Although benign lesions are commoner however there is no doubt that malignant lesions are increasing especially in younger woman. Physical examination, mammography, fine needle aspiration cytology and core needle biopsy are very important in this aspect. ${ }^{3}$

As fine needle aspiration has become a critical component in the investigation of palpable breast lumps, false negative and false positive diagnoses have become a major concern. ${ }^{4}$

However, since FNAC is a cost effective test with a benefit of rapid diagnosis, less patient discomfort; provision of multiple sampling from multiple areas and curative relief in some cases, as in case of aspiration of cyst, FNAC is dependable and beneficial.,

The present study was carried out to know the efficacy of FNAC in giving correct diagnosis and helping the surgeon to plan effective treatment for the patients.

Materials and Methods

Sources of Data: Patients with breast lumps referred from clinical OPD to Cytology section,
Adichunchanagiri Institute of Medical Sciences from October 2011 to September 2013.

Method of Collection of Data: Patients were explained about the procedure and a detailed history was taken. The breast was examined and findings were noted down.

Sample Size: 155 patients were subjected for FNAC and biopsy specimens of those patients who underwent surgical intervention.

FNAC's were performed under aseptic precautions after obtaining consent using a 10cc syringe and 23 gauge needle. Slides were then fixed in Carnoy's fixative and stained for Haematoxylin \& eosin, Pap stain and air dried smears were stained with Giemsa.

Inclusion Criteria: All the patients with breast lumps referred to cytology block were included for the present study

Exclusion Criteria: Cases which didn't have clinical and radiological diagnosis were excluded from the study.

\section{Results}

Out of 155 breast aspirations done in the cytology section, there were 152 female patients and 3 male patients. Majority of the patients belonged to 35-45 years. Benign disease predominated with 128 cases, followed by malignant lesions which were 27 in number (Table 1). 
Table 1: Showing final diagnosis of breast lesions.

\begin{tabular}{|l|c|}
\hline \multicolumn{1}{|c|}{ Breast lesions } & Number of cases \\
\hline Benign breast lesions & 128 \\
\hline Inflammatory lesions & 28 \\
\hline Non-neoplastic lesions & 41 \\
\hline Benign tumors & 59 \\
\hline Malignant breast lesions & 27 \\
\hline Total & 155 \\
\hline
\end{tabular}

Fibroadenoma was the most common breast lesion diagnosed with 50 cases and in the malignant neoplasms, infiltrating ductal carcinoma, NOS was the most common with 19 numbers of cases.

The most common breast lesion encountered is probably fibroadenoma. ${ }^{7}$

Table 2: Showing spectrum of breast lesions

\begin{tabular}{|l|c|c|}
\hline Benign Breast Lesions & & $\mathbf{1 2 8}$ \\
\hline Inflammatory and related & Acute mastitis & 9 \\
\cline { 2 - 3 } lesions & Acute on chronic mastitis & 5 \\
\cline { 2 - 3 } & Breast abscess & 14 \\
\cline { 2 - 3 } & Galactocele & 2 \\
\hline Non neoplastic & Fibrocystic change & 36 \\
\cline { 2 - 3 } & Gynecomastia & 3 \\
\cline { 2 - 3 } & Epithelial hyperplasia & 2 \\
\hline \multirow{4}{*}{ Benign tumors } & Fibroadenoma & 50 \\
\cline { 2 - 3 } & Phyllodes tumor & 4 \\
\cline { 2 - 3 } & Lipoma & 3 \\
\cline { 2 - 3 } & Adenomyoepithelioma & 1 \\
\cline { 2 - 3 } & Haemangioma & 1 \\
\hline Malignant Breast Tumors & & 1 \\
\cline { 2 - 3 } & Infiltrating Ductal carcinoma, NOS & 19 \\
\cline { 2 - 3 } & IDC, Mucinous type & 1 \\
\cline { 2 - 3 } & IDC, Medullary type & 1 \\
\cline { 2 - 3 } & IDC, Papillary type & 15 \\
\cline { 2 - 3 } & Invasive Lobular carcinoma & 3 \\
\cline { 2 - 3 } & Malignant Phyllodes & \\
\hline Total & & \\
\hline
\end{tabular}

\section{Statistical Analysis}

After correlating with clinical and radiological findings a proper diagnosis was given for all the cases. Histopathological correlation was done for cases which had surgical treatment. Using the numbers a statistical analysis was performed to know the efficacy of FNAC.

Biopsy specimens were received for all the 27 cases which were diagnosed as malignant on FNAC. Among those cases which were given as benign on FNAC, specimens were received in 35 cases.

In cytologically benign cases, biopsies obtained all correlated with the cytological diagnosis except one granulomatous breast lesion which was reported as malignant on FNAC turned out to be benign on biopsy (False positive in disease). And in cytologically malignant cases, 2 were reported as benign on FNAC (False negative in disease).

In the case which was cytologically diagnosed as carcinoma breast but turned out to be Granulomatous mastitis, a clinical diagnosis of mastitis was made and the aspiration was grey white thick pus like material. Radiologically mastitis was reported. Smears showed abundant neutrophils, few giant cells and few ductal epithelial cells with atypia. HPE, showed few granulomas and ductal epithelial cells showing mild reactive atypia. (Fig. 1)

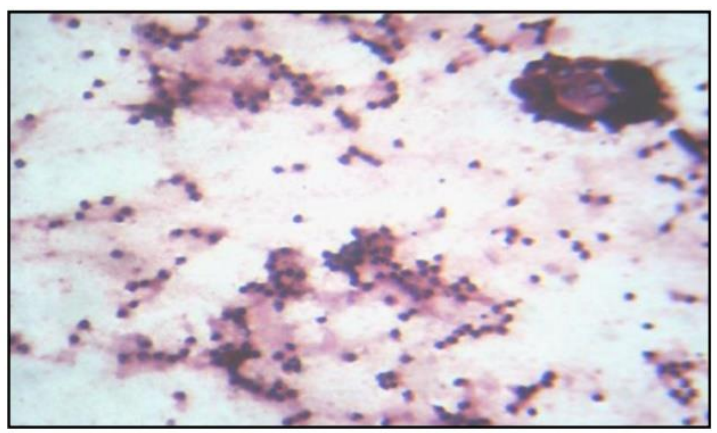

Fig. 1: Cytology: Smears showing neutrophils and ductal epithelial cells showing reactive atypia. 10x. $\mathbf{H} \& \mathbf{E}$

A 56 year old lady with a small hard lump in breast which was clinically suspected of carcinoma and radiologically as dense fibrosis was cytologically diagnosed as sclerosing adenosis and excision biopsy specimen was received which turned out to be infiltrating lobular carcinoma. [Fig. 2 (a), (b)]. 

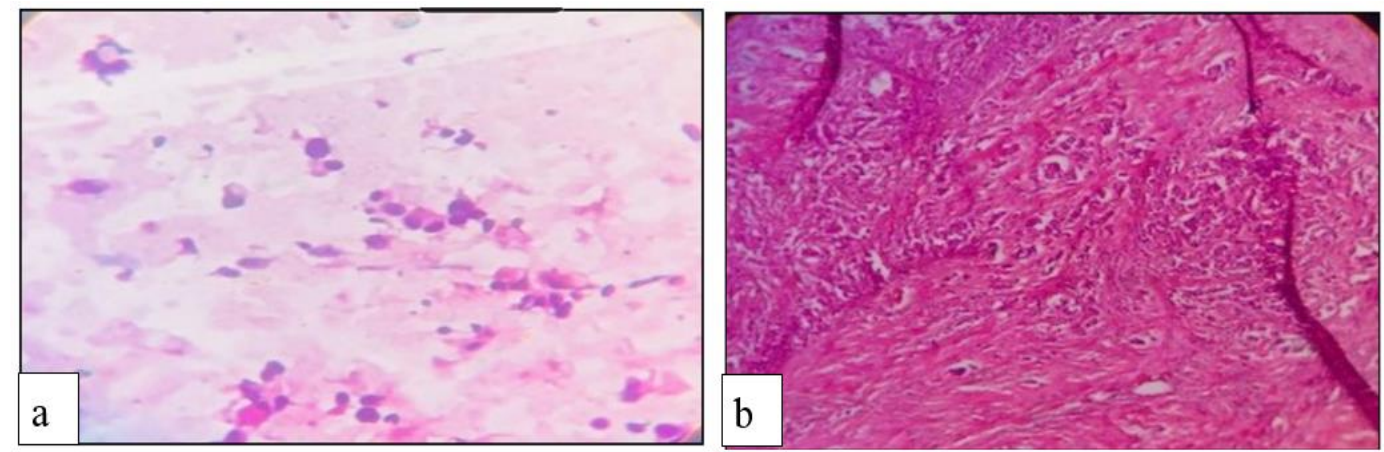

Fig. 2 (a): Cytology: Smear showing scanty cellularity and monomorphic cells in singles. 40x, H \& E.; (b): HP: Abundant fibrous stroma and mildly pleomorphic cells in Indian file pattern. 10x, H \& E

A 45 old lady with a large firm mass in breast suspected as benign phyllodes clinically and malignant phyllodes on radiology a cytologically diagnosis of benign phyllodes was given but turned out be a case of malignant phyllodes on histopathology. [Fig. 3 (a) (b)].
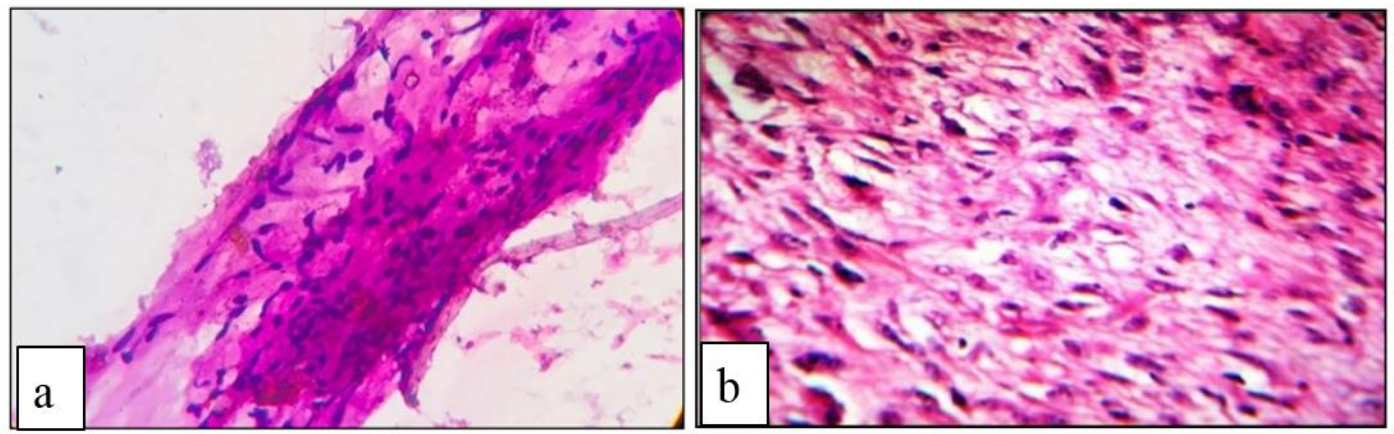

Fig. 3 (a): Cytology: Smear showing large stromal fragments. 40x, H; (b): HP: Section showing leaf like proliferation of the stroma and the lining epithelium showing atypical. 40x, H \& $\mathrm{E}$

Therefore in 3 out of 62 cases, the diagnosis at cytology was not in keeping with histology.

True positives $=27$

False positives $=01$

False negatives $=02$

True negative $=34$

Table 3: Overall statistical analysis

\begin{tabular}{|l|c|}
\hline \multicolumn{1}{|c|}{ Value } & Percentage \\
\hline Sensitivity & $93.1 \%$ \\
\hline Specificity & $97.1 \%$ \\
\hline Positive predictive value & $96.4 \%$ \\
\hline Negative predictive value & $94.4 \%$ \\
\hline
\end{tabular}

\section{Discussion}

The present study confirms the accuracy and clinical utility of FNAC in the diagnosis of benign and malignant breast tumors. A detailed clinical examination to note the consistency and borders of the breast lumps is very much essential along with a supportive radiological findings. Performing FNACs with 22 gauge needles, 1.5 inches length for aspirating is useful since it causes minimal patient discomfort and bleeding. ${ }^{8,9}$
The present study was carried out to know how much FNAC being a simple, cheap and rapid test is beneficial for the patients with breast lumps. A immediate diagnosis as benign lesion offers so much ease to the patients and bystanders. Also diagnosis as malignancy helps the patients to plan for proper and early treatment.

The present study documented 128 cases of benign breast lesions constituting $82.5 \%$ and 27 cases of malignant lesions constituting $17.41 \%$. Fibroadenoma was the most common diagnosis followed by fibrocystic change.

In 128 cases which were cytologically diagnosed as benign, excisional biopsies were obtained for 35 cases. Histopathological examination was carried and discordance was noted for one granulomatous mastitis which was reported as malignant on FNAC.

Among the 27 cases of malignant lesions, most common type diagnosed was IDC seen in 22 cases $(81.4 \%)$, followed by ILC in 3 cases $(11.1 \%)$ and malignant phyllodes tumor in 2 cases $(7.4 \%)$.

In malignant category, 2 cases were reported as benign on FNAC and excisional biopsy showed malignancy.

In the case which was cytologically diagnosed as carcinoma breast but turned out to be Granulomatous 
mastitis, the aspiration was grey white thick pus like material. Smears showed abundant neutrophils, few giant cells and few ductal epithelial cells with atypia. HPE, showed few granulomas and ductal epithelial cells showing mild reactive atypia.

Many studies have concluded that granulomatous mastitis can clinically, radiologically, cytologically mimic carcinoma and a definite diagnosis requires histopathological examination. ${ }^{10}$

Among 3 cases of infiltrating lobular carcinoma, one case was wrongly diagnosed as benign sclerosing adenosis on FNAC. The wrong diagnosis was due to scant cellularity obtained and difficulty in aspiration due to dense fibrosis. However excisional biopsy specimen showed a solid grey white tumor with irregular borders, and microscopy showed malignant epithelial cells in Indian file pattern.

Many studies have reported lobular carcinoma to be the most commonly misdiagnosed type of breast malignancy. ${ }^{11,12}$ Cellularity of the FNAC smears are variable and show monomorphic or mildly pleomorphic cells, mild nuclear atypia and bland chromatin. Many studies have concluded about the variable cellularity obtained during aspiration of ILC cases. ${ }^{13}$

Among the 2 cases of malignant phyllodes, one case was reported as benign phyllodes on FNAC. The aspiration was grey white material. Microscopy showed few ductal epithelial cells in sheets along with many stromal fragments. Excisional biopsy was received and HPE showed malignant fibrous stroma in leaf like pattern with lining epithelium showing dysplastic features.

Main disadvantage of FNAC in phyllodes tumor is that it cannot accurately distinguish between benign, borderline and malignant phyllodes tumor. Mitotic counts, cellularity are subjective and phyllodes tumor constitutes an important grey zone lesion among breast lesions. $^{14}$

Comparison of sensitivity, specificity, positive predictive value and negative predictive value with other published studies in shown in the table below [Table 4].

Table 4: Showing comparison of statistical parameters with other studies

\begin{tabular}{|l|c|c|c|}
\hline \multicolumn{1}{|c|}{ Statistical parameters } & ${\text { Ashwin et } \mathbf{~ a l}^{\mathbf{1 5}}}$ & et al $^{\mathbf{1 6}}$ & Present study \\
\hline Sensitivity & 96.97 & 97.46 & $93.1 \%$ \\
\hline Specificity & 100 & 100 & $97.1 \%$ \\
\hline Positive predictive value & 100 & 100 & $93.1 \%$ \\
\hline Negative predictive value & 98.63 & 100 & $93.1 \%$ \\
\hline
\end{tabular}

\section{Conclusion}

FNAC with its many advantages is still the standard preoperative test for breasts lesions. It offers an immediate rapid diagnosis and benefits the patients with benign lesions who can have minimal surgeries. On the other hand in patients with premalignant lesions it helps patients to recognise the disease before malignant transformation and in malignant category an early diagnosis with simple test can be beneficial.

An occasional false negative and false positive disease makes it mandatory to biopsy in cases where clinico-radiological and cytological diagnosis do not concur and also in cases where the diagnosis of malignancy on cytologic is suspicious.

Lump in the breast is better diagnosed with combined approach of FNAC, clinical diagnosis and radiology.

\section{References}

1. Ochicha, Edno ST, Mohammed AZ, Amin SN. Benign breast lesions in Kano. Nigerian J Surg Research. 2002;4(1):1-5.

2. Muddegowda PH, lingegowda JB, Kurpad R, Konapur PG, Shivarudrappa AS, Subramaniam PM. The value of systematic pattern analysis I FNAC of breast lesions 225 cases with cytohistological correlation. Journal of Cytology. 2011;28((1):13-18.

3. Auger M, Huttner I. Fine needle aspiration cytology of pleomorphic lobular carcinoma of the breast: Comprison with the classic type. Cancer. 1997;18(1):29-32.
4. Bukhari M H, Akhtar Z M. Comparison of accuracy of diagnostic modalties for evaluation of breast cancer with review of literature. Diagnosic cytopathology. 2009;37(6):416-424.

5. Malik Man, Salahuddin O, Azhar M, Dilwar O, Irshad H, Sadia, Salahuddin A. Breast diseases; Spectrum in wah cantt; POF hospital experience. Professional Med J. 2010;17(3):366-372.

6. Bibbo M, Wilbur DC. Comprehensive cytopathology, $3^{\text {rd }}$ edition. Philadelphia Saunders Elsevier, 2008:713715(a);725-775(b).

7. Simsir A, Cangiarella J. Challenging breast lesions: pitfalls and limitations of FNA and the role of core biopsy in specific lesions. Diagn Cytopathol. 2012;40:262-272.

8. Frable WJ. Needle aspiration biopsy: Past, present and future. Hum Pathol. 1989;20:504-517.

9. Rosa M, Mohammadi A, masood S. The value of FNAB in the diagnosis and prognostic assessment of palpable breast lesions. Diagn Cytopathol. 2012;40:26-34.

10. Doughy JC, Wilson CR, Mallon EA. Granulomatous mastitis can mimic breast cancer on clinical, radiological or cytological examination: a cautionary tale. Breast $J$. 2014;13(3):261-262.

11. Rajesh L, dey p, joshi k. FNAC of lobular breast carcinoma. Comparison with other breast lesions. Acta cytol. 2003;47:177-82.

12. Joshi A, Kumar N, Verma K. Diagnostic challenge of lobular carcinoma on aspiration cytology. Diagn Cytopathol. 1998;18:179-83.

13. Abdulla M, Hombal S, Al- Juwaiser A, Stankovich D, Ahmed M. cellularity of lobular carcinoma and its relationship to false negative FAN results. Acta Cytol. 2000;44:625-32. 
14. Shabby NS, Phyllodes tumor. Fine Needle Aspiration Cytology of eight cases. Acta cytol. 1997;41:321-26.

15. Ashwin $\mathrm{P} \mathrm{K}$ et al. Diagnostic utility of FNAC in evaluation of breast masses. International Journal of Recent Scientific Research. 2015;6(8):5827-5831.

16. Anshulekha P, Tapaswini B, Mohanty R C. Utility of FNAC in breast lesions and its correlation with histopathology. 2018;17(2):31-40.

How to cite this article: Susmitha NS, Chaithra H, Anand R. Role of fine needle aspiration cytology ineffective management of patients with breast lesions and histopathological correlation. J Diagn Pathol Oncol. 2018;4(3):150-154. 